TRANSACTIONS OF THE

AMERICAN MATHEMATICAL SOCIETY

Volume 365, Number 6, June 2013, Pages 3287-3307

S 0002-9947(2012)05742-4

Article electronically published on October 4, 2012

\title{
SYMPLECTIC COVARIANCE PROPERTIES FOR SHUBIN AND BORN-JORDAN PSEUDO-DIFFERENTIAL OPERATORS
}

\author{
MAURICE A. DE GOSSON
}

Dedicated to H.G. Feichtinger on his 60th birthday

\begin{abstract}
Among all classes of pseudo-differential operators only the Weyl operators enjoy the property of symplectic covariance with respect to conjugation by elements of the metaplectic group. In this paper we show that there is, however, a weaker form of symplectic covariance for Shubin's $\tau$-dependent operators, in which the intertwiners are no longer metaplectic, but are still invertible non-unitary operators. We also study the case of Born-Jordan operators, which are obtained by averaging the $\tau$-operators over the interval $[0,1]$ (such operators have recently been studied by Boggiatto and his collaborators, and by Toft). We show that covariance still holds for these operators with respect to a subgroup of the metaplectic group.
\end{abstract}

\section{INTRODUCTION}

In the early years of quantum mechanics physicists were confronted with an ordering problem: assume that some quantization process associates to the real variables $x_{j}$ (position) and $p_{j}$ (momentum) two operators $\widehat{X_{j}}$ and $\widehat{P_{j}}$ satisfying the canonical commutation rule $\widehat{X_{j}} \widehat{P_{j}}-\widehat{P_{j}} \widehat{X_{j}}=i \hbar$. Then what should the operator associated to the monomial $x_{j}^{m} p_{j}^{\ell}$ be? The first to give a mathematically motivated answer was Weyl [27]; he was developing his ideas on a group theoretical approach to quantization which led to the prescription

$$
x_{j}^{m} p_{j}^{\ell} \stackrel{\text { Weyl }}{\longrightarrow} \frac{1}{2^{\ell}} \sum_{k=0}^{\ell}\left(\begin{array}{l}
\ell \\
k
\end{array}\right) \widehat{P}_{j}^{\ell-k} \widehat{X}_{j}^{m}{\widehat{P_{j}}}^{k} .
$$

It turns out that the Weyl ordering is a particular case of the more general " $\tau$ ordering": for any real number $\tau$ one defines

$$
x_{j}^{m} p_{j}^{\ell} \stackrel{\tau}{\longrightarrow} \sum_{k=0}^{\ell}\left(\begin{array}{l}
\ell \\
k
\end{array}\right)(1-\tau)^{k} \tau^{\ell-k}{\widehat{P_{j}}}^{\ell-k} \widehat{X}_{j}^{m} \widehat{P}_{j}^{k},
$$

Received by the editors May 6, 2011 and, in revised form, November 5, 2011.

2010 Mathematics Subject Classification. Primary 47G30; Secondary 35Q40, 65P10, 35S05, $42 \mathrm{~B} 10$.

Key words and phrases. Pseudo-differential operators, metaplectic group, symplectic covariance. 
which reduces to Weyl's prescription when $\tau=\frac{1}{2}$. From now on we will assume that $\widehat{X_{j}} f=x_{j} f$ and $\widehat{P_{j}} f=(1 / 2 \pi i) \partial_{x_{j}} f$. The $\tau$-ordering (1.2) is itself a particular case of the Shubin pseudo-differential calculus (Shubin 23]): given a symbol $a$ the $\tau$-pseudo-differential operator $A_{\tau}=\mathrm{Op}_{\tau}(a)$ is formally defined by

$$
A_{\tau} f(x)=\iint e^{2 \pi i p(x-y)} a(\tau x+(1-\tau) y, p) f(y) d p d y
$$

For $\tau=\frac{1}{2}$ we recover the well-known Weyl correspondence: $A_{1 / 2}=A_{\mathrm{Weyl}}=\widehat{A}$, where

$$
\widehat{A} f(x)=\iint e^{2 \pi i p(x-y)} a\left(\frac{1}{2}(x+y), p\right) f(y) d p d y .
$$

Using Schwartz's kernel theorem it is not difficult to show that for every continuous linear operator $A: \mathcal{S}\left(\mathbb{R}^{n}\right) \longrightarrow \mathcal{S}^{\prime}\left(\mathbb{R}^{n}\right)$ and for every $\tau \in \mathbb{R}$ there exists $a \in \mathcal{S}^{\prime}\left(\mathbb{R}^{2 n}\right)$ such that $A=\mathrm{Op}_{\tau}(a)$; the $\tau$-operators are thus of a very general nature. Now, it is (reasonably) well known (Stein [24, Wong 28]) that among all $\tau$-operators only the Weyl operators enjoy a symmetry property known as "symplectic covariance with respect to the metaplectic group":

$$
\text { If } \mathrm{Op}_{\tau}(a \circ S)=\widehat{S} \mathrm{Op}_{\tau}(a) \widehat{S}^{-1} \text { for every } \widehat{S} \in \mathrm{Mp}(2 n, \mathbb{R}) \text {, then } \tau=\frac{1}{2} .
$$

Here $\operatorname{Mp}(2 n, \mathbb{R})$ is the metaplectic group and $S$ the projection of $\widehat{S} \in \operatorname{Mp}(2 n, \mathbb{R})$ on the symplectic group $\operatorname{Sp}(2 n, \mathbb{R})$. This property is very important in Hörmander's approach to Weyl's calculus in Chapter 18 (Theorem 18.5.9) of [19].

Symplectic covariance in the sense above is thus really a characteristic property of Weyl pseudo-differential calculus. In fact, one shows more generally that:

Let $a \longmapsto \mathrm{Op}(a)$ be a linear mapping from $\mathcal{S}^{\prime}\left(\mathbb{R}^{2 n}\right)$ to the space of linear operators that is continuous in the topology of $\mathcal{S}^{\prime}\left(\mathbb{R}^{2 n}\right)$. Assume that: (i) if $a=a(x), a \in L^{\infty}\left(\mathbb{R}^{n}\right)$, then $\mathrm{Op}(a)$ is multiplication by $a(x)$; (ii) if $S \in \mathrm{Sp}(2 n, \mathbb{R})$, then $\mathrm{Op}(a \circ S)=\widehat{S} \mathrm{Op}(a) \widehat{S}^{-1}$. Then $a \longmapsto \mathrm{Op}(a)$ is the Weyl correspondence

(Stein [24], §12.7, Wong, Chapter 30), so the property of symplectic covariance really singles out Weyl operators among all possible "quantization schemes".

The principal aim of this paper is to report on the fact that there exists a weaker form of symplectic covariance for $\tau$-operators which reduces to the case above when $\tau=\frac{1}{2}$. In fact, we will show in Proposition 3 that to each $S \in \operatorname{Sp}(2 n, \mathbb{R})$ with $\operatorname{det}(S-I) \neq 0$ we can associate an invertible operator $R_{\tau}(S): \mathcal{S}\left(\mathbb{R}^{n}\right) \longrightarrow \mathcal{S}\left(\mathbb{R}^{n}\right)$ such that

$$
R_{\tau}(S) \mathrm{Op}_{\tau}(a)=\mathrm{Op}_{\tau}(a \circ S) R_{\tau}(S) .
$$

The operator $R_{\tau}(S)$ is explicitly given by the simple looking expression

$$
R_{\tau}(S)=\sqrt{|\operatorname{det}(S-I)|} \int \widehat{T}_{\tau}(S z) \widehat{T}_{\tau}(-z) d z,
$$

where $\widehat{T}_{\tau}(z)$ is up to a factor depending on $\tau$ and $z$ to the Heisenberg operator $\widehat{T}(z)$ familiar from harmonic analysis. The twisted symbol of $R_{\tau}(S)$, viewed as 
$\tau$-pseudo-differential operator, is given by the formula

$$
r_{\sigma}(z)=\frac{1}{\sqrt{|\operatorname{det}(S-I)|}} e^{i \pi M(S) z^{2}},
$$

where $M(S)$ is the symplectic Cayley transform of $S$.

The operator $R_{\tau}(S)$ is generally not unitary, and thus does not generate a copy of $\operatorname{Mp}(2 n, \mathbb{R})$. However it does generate a group $\operatorname{Mp}_{\tau}(2 n, \mathbb{R})$ of invertible operators $R_{\tau}^{\nu}(S)$ ( $S$ an arbitrary element of $\left.\operatorname{Sp}(2 n, \mathbb{R})\right)$ which is, as we will see, algebraically isomorphic to the metaplectic group $\operatorname{Mp}(2 n, \mathbb{R})$. This group is actually a group of intertwiners for $\tau$-operators, in the sense that we have the property

$$
R_{\tau}^{\nu}(S) \mathrm{Op}_{\tau}(a)=\mathrm{Op}_{\tau}(a \circ S) R_{\tau}^{\nu}(S)
$$

which truly generalizes the usual symplectic covariance with respect to the metaplectic group which only holds for Weyl operators.

We will also study symplectic covariance properties for Born-Jordan pseudodifferential operators. These operators were very recently introduced in de Gosson and Luef [17, where it was remarked that the constructions of Boggiatto and his collaborators [1, 2, 3] are related to a quantization procedure going back to Born and Jordan 4 and historically anterior to the work of Weyl [27]. Born and Jordan's quantization is based on the prescription

$$
x^{m} p^{\ell} \stackrel{\mathrm{BJ}}{\longrightarrow} \frac{1}{\ell+1} \sum_{k=0}^{\ell} \widehat{P}^{\ell-k} \widehat{X}^{m} \widehat{P}^{k} ;
$$

an elementary calculation shows that this correspondence is obtained by averaging the $\tau$-ordering (1.2) over the interval $[0,1]$. This suggests that one define more generally the Born-Jordan pseudo-differential operator with symbol $a$ by the formula

$$
A_{\mathrm{BJ}}=\int_{0}^{1} A_{\tau} d \tau
$$

We will see that while one cannot expect any formula of the type (1.5), Born-Jordan operators are nevertheless symplectically covariant with respect to a subgroup of $\operatorname{Mp}(2 n, \mathbb{R})$, containing the Fourier transform as well as unitary rescalings.

A topic we have not included in this paper is the study of the properties of these operators on Feichtinger's [6, 7] modulation spaces. It turns out that there is a close relationship between Shubin operators and Shubin classes, which are in turn part of the family of modulation spaces ( $L^{2}$-spaces with radial symmetric weights). We will come back to these properties in future work.

Notation 1. The Euclidean scalar product of two vectors $u$ and $v$ on $\mathbb{R}^{m}$ is denoted indifferently by $u \cdot v$ or by $u v$. When $X$ is a symmetric matrix we will often write $X u^{2}$ for $X u \cdot u$. The standard symplectic form $\sigma$ on $\mathbb{R}^{n} \times \mathbb{R}^{n} \equiv \mathbb{R}^{2 n}$ is defined by $\sigma\left(z, z^{\prime}\right)=p x^{\prime}-p^{\prime} x$. If $z=(x, p), z^{\prime}=\left(x^{\prime}, p^{\prime}\right)$, the corresponding symplectic group is $\operatorname{Sp}(2 n, \mathbb{R})$. We denote by $\mathcal{S}\left(\mathbb{R}^{n}\right)$ the Schwartz space of rapidly decreasing functions on $\mathbb{R}^{n}$ and by $\mathcal{S}^{\prime}\left(\mathbb{R}^{n}\right)$ its dual (the tempered distributions). The normalizations we use correspond to those familiar with the theory of pseudo-differential operators and harmonic analysis. For instance, the Fourier transform of $f \in \mathcal{S}\left(\mathbb{R}^{n}\right)$ is

$$
F f(x)=\int e^{-2 \pi i x x^{\prime}} f\left(x^{\prime}\right) d x^{\prime}
$$

(it corresponds to the choice $\hbar=1 / 2 \pi$ in the quantum-mechanical literature). 


\section{The Shubin CAlculus}

\subsection{Definitions and main properties.}

2.1.1. The pseudo-differential operators $A_{\tau}$. The $\tau$-pseudo-differential operator $A_{\tau}$ $=\mathrm{Op}_{\tau}(a)$ with symbol $a \in \mathcal{S}^{\prime}\left(\mathbb{R}^{n}\right)$ is, by definition, the operator with distributional kernel

$$
K_{\tau}(x, y)=F_{2}^{-1}[a(\tau x+(1-\tau) y, \cdot)](x-y),
$$

where $F_{2}^{-1}$ is the inverse Fourier transform in the second set of variables. We can thus formally write (Shubin [23])

$$
A_{\tau} f(x)=\iint e^{2 \pi i p(x-y)} a(\tau x+(1-\tau) y, p) f(y) d p d y .
$$

One easily verifies by using this expression that the (formal) adjoint of $A_{\tau}=\mathrm{Op}_{\tau}(a)$ is given by

$$
\mathrm{Op}_{\tau}(a)^{*}=\mathrm{Op}_{1-\tau}(\bar{a})
$$

2.1.2. The operators $\widehat{T}_{\tau}(z)$. Let $\widehat{T}\left(z_{0}\right)$ be the Heisenberg operator: it is defined for $f \in \mathcal{S}^{\prime}\left(\mathbb{R}^{n}\right)$ by

$$
\widehat{T}\left(z_{0}\right) f(x)=e^{2 \pi i\left(p_{0} x-\frac{1}{2} p_{0} x_{0}\right)} f\left(x-x_{0}\right),
$$

where $z_{0}=\left(x_{0}, p_{0}\right)$. Let $\tau$ be a real parameter and set, more generally,

$$
\widehat{T}_{\tau}\left(z_{0}\right) f(x)=e^{2 \pi i\left(p_{0} x-(1-\tau) p_{0} x_{0}\right)} f\left(x-x_{0}\right) ;
$$

that is, equivalently,

$$
\widehat{T}_{\tau}\left(z_{0}\right)=e^{i \pi(2 \tau-1) p_{0} x_{0}} \widehat{T}\left(z_{0}\right) .
$$

We have $\widehat{T}_{1 / 2}\left(z_{0}\right)=\widehat{T}\left(z_{0}\right)$, and

$$
\widehat{T}_{\tau}\left(z_{0}\right)^{-1}=\widehat{T}_{1-\tau}\left(-z_{0}\right) .
$$

It is immediate to check the following relations:

$$
\begin{aligned}
\widehat{T}_{\tau}\left(z_{0}\right) \widehat{T}_{\tau}\left(z_{1}\right) & =e^{2 \pi i \sigma_{\tau}\left(z_{0}, z_{1}\right)} \widehat{T}_{\tau}\left(z_{1}\right) \widehat{T}_{\tau}\left(z_{0}\right), \\
\widehat{T}_{\tau}\left(z_{0}+z_{1}\right) & =e^{-i \pi \sigma_{\tau}\left(z_{0}, z_{1}\right)} \widehat{T}_{\tau}\left(z_{0}\right) \widehat{T}_{\tau}\left(z_{1}\right),
\end{aligned}
$$

where

$$
\sigma_{\tau}\left(z_{0}, z_{1}\right)=2(1-\tau) p_{0} x_{1}-2 \tau p_{1} x_{0} .
$$

Note that $\sigma_{\tau}$ is a bilinear form, but not in general a symplectic form (it fails to be antisymmetric if $\tau \neq \frac{1}{2}$ ).

For many purposes it is useful to write formula (2.2) in terms of the operators $\widehat{T}_{\tau}(z)$ :

$$
A_{\tau} f=\mathrm{Op}_{\tau}(a) f=\int a_{\sigma}(z) \widehat{T}_{\tau}(z) f d z,
$$

where $a_{\sigma}$ is the symplectic Fourier transform of $a$; that is,

$$
a_{\sigma}(z)=\int e^{-2 \pi i \sigma\left(z, z^{\prime}\right)} a\left(z^{\prime}\right) d z^{\prime} .
$$


Following the usage in the theory of Weyl operators, we will call $a_{\sigma}$ the "twisted symbol of $A_{\tau}$ ". The distributional kernel of $A_{\tau}$ can then be written

$$
K_{\tau}(x, y)=F_{2}^{-1}\left[a_{\sigma}(x-y, \cdot)\right](\tau x+(1-\tau) y) ;
$$

it is often more suitable for calculations than formula (2.1) above.

2.1.3. A composition formula. The $\tau$-operators can be composed in exactly the same way as usual Weyl operators:

Proposition 1. Let $A_{\tau}=\mathrm{Op}_{\tau}(a)$ and $B_{\tau}=\mathrm{Op}_{\tau}(b)$ with $a, b \in \mathcal{S}\left(\mathbb{R}^{2 n}\right)$ (or, more generally, $a \in \mathcal{S}^{\prime}\left(\mathbb{R}^{2 n}\right)$ and $b \in \mathcal{S}\left(\mathbb{R}^{2 n}\right)$ ). We have $A_{\tau} B_{\tau}=C_{\tau}=\mathrm{Op}_{\tau}(c)$ with

$$
c_{\sigma}(z)=\int e^{i \pi \sigma_{\tau}\left(z-z^{\prime}, z^{\prime}\right)} a_{\sigma}\left(z-z^{\prime}\right) b_{\sigma}\left(z^{\prime}\right) d z^{\prime}
$$

where the integral should be viewed as a distributional bracket when a $\in \mathcal{S}^{\prime}\left(\mathbb{R}^{2 n}\right)$ and $b \in \mathcal{S}\left(\mathbb{R}^{2 n}\right)$.

Proof. Writing

$$
A_{\tau}=\int a_{\sigma}(z) \widehat{T}_{\tau}(z) d z \text { and } \quad B_{\tau}=\int b_{\sigma}(z) \widehat{T}_{\tau}(z) d z .
$$

we have

$$
A_{\tau} B_{\tau}=\iint a_{\sigma}\left(z_{0}\right) b_{\sigma}\left(z_{1}\right) \widehat{T}_{\tau}\left(z_{0}\right) \widehat{T}_{\tau}\left(z_{1}\right) d z_{0} d z_{1},
$$

and hence, using formula (2.9),

$$
A_{\tau} B_{\tau}=\iint e^{i \pi \sigma_{\tau}\left(z_{0}, z_{1}\right)} a_{\sigma}\left(z_{0}\right) b_{\sigma}\left(z_{1}\right) \widehat{T}_{\tau}\left(z_{0}+z_{1}\right) d z_{0} d z_{1} .
$$

The composition formula (2.12) follows by making the change of variables $z=$ $z_{0}+z_{1}, z^{\prime}=z_{1}$.

2.2. The $\tau$-Wigner transform. Boggiatto and his collaborators [1, 2, 3] have recently studied a $\tau$-dependent Wigner transform $W_{\tau}(f, g)$ (which has independently been considered by Toft [25]). This transform is related to the Shubin $\tau$-pseudodifferential calculus. Averaging $W_{\tau}$ over $\tau$ in the interval $[0,1]$ leads to an element of the Cohen class, i.e. to a transform of the type $Q(f, g)=W_{\tau}(f, g) * \theta$ where $\theta \in \mathcal{S}^{\prime}\left(\mathbb{R}^{2 n}\right)$.

The following result relates the operator $A_{\tau}$ to the $\tau$-Wigner transform:

Proposition 2. Let $f, g \in \mathcal{S}\left(\mathbb{R}^{n}\right)$. We have the formula

$$
\left(A_{\tau} f \mid g\right)_{L^{2}}=\left\langle a, W_{\tau}(f, g)\right\rangle
$$

where $W_{\tau}(f, g)$ is the $\tau$-dependent cross-Wigner transform of $(f, g)$ defined by

$$
W_{\tau}(f, g)(z)=\int e^{-2 \pi i y p} f(x+\tau y) \overline{g(x-(1-\tau) y)} d y .
$$

Proof. We have

$$
\left\langle a, W_{\tau}(f, g)\right\rangle=\int e^{-2 \pi i y p} a(z) f(x+\tau y) \overline{g(x-(1-\tau) y)} d y d p d x .
$$

Setting $y^{\prime}=x+\tau y=, x^{\prime}=x-(1-\tau) y$ we get

$$
\left\langle a, W_{\tau}(f, g)\right\rangle=\int e^{-2 \pi i\left(x^{\prime}-y^{\prime}\right) p} a\left((1-\tau) x^{\prime}+\tau y^{\prime}, p\right) f\left(y^{\prime}\right) \overline{g\left(x^{\prime}\right)} d y^{\prime} d p d x^{\prime},
$$

and hence the equality (2.14) in view of (2.2). 
Formula (2.14) yields an alternative definition of the operator $A_{\tau} f$ for an arbitrary symbol $a \in \mathcal{S}^{\prime}\left(\mathbb{R}^{n}\right)$ and $f \in \mathcal{S}\left(\mathbb{R}^{n}\right)$ : choose $g \in \mathcal{S}\left(\mathbb{R}^{n}\right)$; then $W_{\tau}(f, g) \in \mathcal{S}\left(\mathbb{R}^{2 n}\right)$ and the distributional bracket $\left\langle a, W_{\tau}(f, g)\right\rangle$ is thus defined; by definition $A_{\tau}$ is the continuous operator $\mathcal{S}\left(\mathbb{R}^{n}\right) \longrightarrow \mathcal{S}^{\prime}\left(\mathbb{R}^{n}\right)$ defined by the right-hand side of (2.14).

Remark 2.1. The result above is closely related to (1.4) (and to some extent to Proposition 4.11) in Toft [25].

We notice that the $\tau$-dependent Wigner transform $W_{\tau} \psi=W_{\tau}(f, g)$ satisfies the same marginal properties as the ordinary Wigner transform: for every $f \in$ $L^{1}\left(\mathbb{R}^{n}\right) \cap L^{2}\left(\mathbb{R}^{n}\right)$ we have

$$
\int W_{\tau} f(x, p) d p=|f(x)|^{2}, \int W_{\tau} f(x, p) d x=|\widehat{f}(p)|^{2}
$$

(see Boggiatto et al. [1]).

In the case $\tau=1$ the transform $W_{\tau}$ reduces to the Rihaczek distribution

$$
\mathcal{R}(f, g)(z)=e^{2 \pi i p \cdot x} \widehat{f}(x) \overline{g(x)}
$$

for $f, g \in L^{2}\left(\mathbb{R}^{n}\right)$. When $\tau=0$ we get the so-called dual Rihaczek distribution

$$
\mathcal{R}^{*}(f, g)(z)=e^{-2 \pi i p \cdot x} f(x) \overline{\widehat{g}(x)} .
$$

\section{Symplectic covariance in Shubin calculus}

\subsection{A class of intertwining operators.}

3.1.1. The symplectic Cayley transform. We will use the following notation:

$$
\begin{aligned}
\operatorname{Sp}_{(0)}(2 n, \mathbb{R}) & =\{S \in \operatorname{Sp}(2 n, \mathbb{R}): \operatorname{det}(S-I) \neq 0\}, \\
\operatorname{Sym}_{(0)}(2 n, \mathbb{R}) & =\left\{M \in \operatorname{Sym}(2 n, \mathbb{R}): \operatorname{det}\left(M-\frac{1}{2} J\right) \neq 0\right\} .
\end{aligned}
$$

Let $S \in \operatorname{Sp}_{(0)}(2 n, \mathbb{R})$. By definition the symplectic Cayley transform (introduced in de Gosson [12, 13, 14, 15, 16]) of $S$ is the symmetric matrix given by

$$
M(S)=\frac{1}{2} J(S+I)(S-I)^{-1}
$$

(the symmetry of $M(S)$ is readily verified using the relation $S^{T} J S=S J S^{T}=J$, which is equivalent to $S \in \operatorname{Sp}(2 n, \mathbb{R}))$. It is clear that the mapping $M(\cdot)$ is a bijection $\operatorname{Sp}_{(0)}(2 n, \mathbb{R}) \longrightarrow \operatorname{Sym}_{(0)}(2 n, \mathbb{R})$ and that the inverse of that bijection is given by

$$
S=\left(M-\frac{1}{2} J\right)^{-1}\left(M+\frac{1}{2} J\right)
$$

We have the properties

$$
M\left(S^{-1}\right)=-M(S)
$$

and, when in addition $S^{\prime}, S S^{\prime} \in \operatorname{Sp}_{(0)}(2 n, \mathbb{R})$,

$$
M\left(S S^{\prime}\right)=M(S)+\left(S^{T}-I\right)^{-1} J\left(M(S)+M\left(S^{\prime}\right)\right)^{-1} J(S-I)^{-1} .
$$


3.1.2. The intertwining operators $R_{\tau}(S)$. We will need the following well-known generalization of the Fresnel formula (see e.g. Folland [8], Appendix A): let $X$ be a real invertible matrix of dimension $m$; then

$$
\int e^{-2 \pi i u v} e^{i \pi X v^{2}} d v=|\operatorname{det} X|^{-1 / 2} e^{\frac{i \pi}{4} \operatorname{sign} X} e^{-i \pi X^{-1} u^{2}},
$$

where $\operatorname{sign} X$ is the difference between the number of $>0$ and $<0$ eigenvalues of $X$. Using this formula and the two propositions above we set out to study the operators

$$
R_{\tau}(S)=\sqrt{|\operatorname{det}(S-I)|} \int \widehat{T}_{\tau}(S z) \widehat{T}_{\tau}(-z) d z
$$

defined for $S \in \operatorname{Sp}_{(0)}(2 n, \mathbb{R})$.

Proposition 3. (i) Let $S \in \operatorname{Sp}_{(0)}(2 n, \mathbb{R})$. The operator $R_{\tau}(S)$ is a continuous mapping $\mathcal{S}\left(\mathbb{R}^{n}\right) \longrightarrow \mathcal{S}\left(\mathbb{R}^{n}\right)$ satisfying

$$
R_{\tau}(S) \widehat{T}_{\tau}(z)=\widehat{T}_{\tau}(S z) R_{\tau}(S),
$$

and we have, for $a \in \mathcal{S}^{\prime}\left(\mathbb{R}^{2 n}\right)$,

$$
R_{\tau}(S) \mathrm{Op}_{\tau}(a)=\mathrm{Op}_{\tau}(a \circ S) R_{\tau}(S) .
$$

(ii) Let $S, S^{\prime} \in \operatorname{Sp}_{(0)}(2 n, \mathbb{R})$ and assume that $S S^{\prime} \in \operatorname{Sp}_{(0)}(2 n, \mathbb{R})$ as well. We have

$$
R_{\tau}\left(S S^{\prime}\right)=e^{i \frac{\pi}{4} \operatorname{sign} M\left(S S^{\prime}\right)} R_{\tau}(S) R_{\tau}\left(S^{\prime}\right) .
$$

(iii) We also have

$$
R_{\tau}\left(S^{-1}\right)=R_{\tau}(S)^{-1}=R_{1-\tau}(S)^{*} .
$$

Proof. (i) It is equivalent to show that the operators

$$
\Gamma_{\tau}(S)=\int \widehat{T}_{\tau}(S z) \widehat{T}_{\tau}(-z) d z
$$

are such that $\Gamma_{\tau}(S) \widehat{T}_{\tau}(z)=\widehat{T}_{\tau}(S z) \Gamma_{\tau}(S)$. Let $f \in \mathcal{S}\left(\mathbb{R}^{n}\right)$; in view of formula (2.9) we have

$$
\Gamma_{\tau}(S) f=\int e^{i \pi \sigma(S z, z)} \widehat{T}_{\tau}((S-I) z) f d z .
$$

Since $S-I$ is a linear automorphism, $\widehat{T}_{\tau}((S-I) z): \mathcal{S}\left(\mathbb{R}^{n}\right) \longrightarrow \mathcal{S}\left(\mathbb{R}^{n}\right)$; hence $\Gamma_{\tau}(S) f \in \mathcal{S}\left(\mathbb{R}^{n}\right)$. The continuity of $\Gamma_{\tau}(S)$ is straightforward to verify. Set

$$
\begin{aligned}
& F\left(z, z_{0}\right)=\widehat{T}_{\tau}(S z) \widehat{T}_{\tau}(-z) \widehat{T}_{\tau}\left(z_{0}\right), \\
& G\left(z, z_{0}\right)=\widehat{T}_{\tau}\left(S z_{0}\right) \widehat{T}_{\tau}(S z) \widehat{T}_{\tau}(-z) .
\end{aligned}
$$

By repeated use of formula (2.9) we obtain

$$
\begin{aligned}
& F\left(z, z_{0}\right)=e^{-i \pi \sigma\left(S z-z_{0}, z-z_{0}\right)} \widehat{T}_{\tau}\left((S-I) z+z_{0}\right), \\
& G\left(z, z_{0}\right)=e^{-i \pi \sigma\left((S-I) z_{0}+S z_{0}, z\right)} \widehat{T}_{\tau}\left((S-I) z+S z_{0}\right) ;
\end{aligned}
$$

hence $G\left(z-z_{0}, z_{0}\right)=F\left(z, z_{0}\right)$. It follows that $\int F\left(z, z_{0}\right) d z=\int G\left(z, z_{0}\right) d z$, and hence the equality (3.7). That the operators $R_{\tau}(S)$ satisfy the intertwining relation (3.8) follows by using definition (2.10) of $\mathrm{Op}_{\tau}(a)$. 
(ii) (Cf. the proof of Proposition 4.2 in de Gosson 14.) For brevity we write $M=M(S), M^{\prime}=M\left(S^{\prime}\right)$. In view of the composition formula (2.12) the twisted symbol $c_{\sigma}$ of $R_{\tau}(S) R_{\tau}\left(S^{\prime}\right)$ is

$$
c_{\sigma}(z)=K \int e^{i \pi\left[\sigma\left(z, z^{\prime}\right)+\Phi\left(z, z^{\prime}\right)\right]} d z^{\prime},
$$

where the constant $K$ and the phase $\Phi$ are given by

$$
\begin{aligned}
K & =\left|\operatorname{det}(S-I)\left(S^{\prime}-I\right)\right|^{-1 / 2}, \\
\Phi\left(z, z^{\prime}\right) & =M z^{2}-2 M z \cdot z^{\prime}+\left(M+M^{\prime}\right) z^{\prime 2} .
\end{aligned}
$$

A straightforward calculation shows that

$$
\sigma\left(z, z^{\prime}\right)-2 M z \cdot z^{\prime}=-2 J(S-I)^{-1} z \cdot z^{\prime}
$$

hence

$$
\sigma\left(z, z^{\prime}\right)+\Phi\left(z, z^{\prime}\right)=-2 J(S-I)^{-1} z \cdot z^{\prime}+M z^{2}+\left(M+M^{\prime}\right) z^{\prime 2} .
$$

It follows that

$$
c_{\sigma}(z)=K e^{i \pi M z^{2}} \int e^{-2 \pi i J(S-I)^{-1} z \cdot z^{\prime}} e^{i \pi\left(M+M^{\prime}\right) z^{\prime 2}} d z^{\prime} .
$$

Applying the Fresnel formula (3.5) with $X=M+M^{\prime}$ to (3.12) and replacing $K$ with its value we get

$$
c_{\sigma}(z)=\left|\operatorname{det}\left[\left(M+M^{\prime}\right)(S-I)\left(S^{\prime}-I\right)\right]\right|^{-1 / 2} e^{\frac{i \pi}{4} \operatorname{sign}\left(M+M^{\prime}\right)} e^{2 \pi i \Theta(z)},
$$

where the phase $\Theta$ is given by

$$
\begin{aligned}
\Theta(z) & =\left[M+\left(S^{T}-I\right)^{-1} J\left(M+M^{\prime}\right)^{-1} J(S-I)^{-1}\right] z^{2} \\
& =M\left(S S^{\prime}\right) z^{2}
\end{aligned}
$$

(the second equality in view of formula (3.4)). Noting that by definition of the symplectic Cayley transform we have

$$
M+M^{\prime}=J\left(I+(S-I)^{-1}+\left(S^{\prime}-I\right)^{-1}\right),
$$

it follows, again using property (3.4), that

$$
\begin{aligned}
\operatorname{det}\left[\left(M+M^{\prime}\right)(S-I)\left(S^{\prime}-I\right)\right] & =\operatorname{det}\left[(S-I)\left(M+M^{\prime}\right)\left(S^{\prime}-I\right)\right] \\
& =\operatorname{det}\left(S S^{\prime}-I\right),
\end{aligned}
$$

which concludes the proof of the product formula (3.9).

(iii) Let us first show that $R_{\tau}\left(S^{-1}\right)=R_{\tau}(S)^{-1}$. Let $c$ be the symbol of $C=R_{\tau}(S) R_{\tau}\left(S^{-1}\right)$; we claim that $c_{\sigma}(z)=\delta(z)$, and hence $C=I$. Noting that $\operatorname{det}\left(S^{-1}-I\right)=\operatorname{det}(S-I) \neq 0$, formula (3.11) in the proof of part (ii) shows that we have

$$
c_{\sigma}(z)=L e^{i \pi M z^{2}} \int e^{-2 \pi i J(S-I)^{-1} z \cdot z^{\prime}} e^{i \pi\left(M+M\left(S^{-1}\right)\right) z^{\prime 2}} d z^{\prime},
$$

where $L=|\operatorname{det}(S-I)|^{-1}$. Since $M\left(S^{-1}\right)=-M$ we have, setting $z^{\prime \prime}=\left(S^{T}-\right.$ $I)^{-1} J z^{\prime}$

$$
\begin{aligned}
c_{\sigma}(z) & =\frac{e^{i \pi M z^{2}}}{|\operatorname{det}(S-I)|} \int e^{-2 \pi i J(S-I)^{-1} z \cdot z^{\prime}} d z^{\prime} \\
& =e^{i \pi M z^{2}} \int e^{2 \pi i z z^{\prime \prime}} d z^{\prime \prime} .
\end{aligned}
$$


Hence $c_{\sigma}(z)=\delta(z)$ by the Fourier inversion formula, which proves that $C=I$. Let us finally show that $R_{\tau}\left(S^{-1}\right)=R_{1-\tau}(S)^{*}$. We have

$$
\begin{aligned}
R_{\tau}\left(S^{-1}\right) & =\frac{1}{\sqrt{\left|\operatorname{det}\left(S^{-1}-I\right)\right|}} \int e^{i \pi M\left(S^{-1}\right) z^{2}} \widehat{T}_{\tau}(z) d z \\
& =\frac{1}{\sqrt{|\operatorname{det}(S-I)|}} \int e^{-i \pi M(S) z^{2}} \widehat{T}_{\tau}(z) d z .
\end{aligned}
$$

Hence, using formula (2.3) for the adjoint of a $\tau$-operator,

$$
R_{\tau}\left(S^{-1}\right)^{*}=\frac{1}{\sqrt{|\operatorname{det}(S-I)|}} \int e^{i \pi M(S) z^{2}} \widehat{T}_{1-\tau}(z) d z=R_{1-\tau}(S),
$$

which is the same thing as $R_{\tau}\left(S^{-1}\right)=R_{1-\tau}(S)^{*}$.

Notice that formula (3.10) shows that the operators $R_{\tau}(S)$ are unitary if and only if $\tau=\frac{1}{2}$ (the Weyl case (1.4)). See de Gosson [12, 13, 14, 15, 16] for a detailed discussion of this and related properties.

3.1.3. Application to the $\tau$-Wigner function. The usual cross-Wigner function $W(f, g)$ has the following well-known (and very useful) property of symplectic covariance: for all $f, g \in \mathcal{S}\left(\mathbb{R}^{n}\right)$ and $S \in \operatorname{Sp}(2 n, \mathbb{R})$ we have

$$
W(\widehat{S} f, \widehat{S} g)(z)=W(f, g)\left(S^{-1} z\right),
$$

where $\widehat{S} \in \operatorname{Mp}(2 n, \mathbb{R})$ is any of the two metaplectic operators which cover $S$. In the $\tau$-dependent case this result must be modified as follows:

Proposition 4. Let $S \in \operatorname{Sp}_{(0)}(2 n, \mathbb{R})$ and $f, g \in \mathcal{S}\left(\mathbb{R}^{n}\right)$. We have

$$
W_{\tau}\left(R_{\tau}(S) f, R_{1-\tau}(S) g\right)(z)=W_{\tau}(f, g)\left(S^{-1} z\right) .
$$

Proof. Let $A_{\tau}=\mathrm{Op}_{\tau}(a)$. Recall that $\left(\mathrm{Op}_{\tau}(a) f \mid g\right)_{L^{2}}=\left\langle a \mid W_{\tau}(f, g)\right\rangle$ (formula (2.14) $)$. In view of the second equality (3.10) we have

$$
\begin{aligned}
\left(R_{\tau}(S) \mathrm{Op}_{\tau}(a) f \mid R_{1-\tau}(S) g\right)_{L^{2}} & =\left(R_{1-\tau}(S)^{*} R_{\tau}(S) \underset{\tau}{\mathrm{Op}}(a) f \mid g\right)_{L^{2}} \\
& =\left(\mathrm{Op}_{\tau}(a) f \mid g\right)_{L^{2}} \\
& =\left\langle a \mid W_{\tau}(f, g)\right\rangle .
\end{aligned}
$$

On the other hand, using the intertwining property (3.8), we have

$$
\begin{aligned}
\left(R_{\tau}(S) \mathrm{Op}_{\tau}(a) f \mid R_{1-\tau}(S) g\right)_{L^{2}} & =\left(\mathrm{Op}_{\tau}(a \circ S) R_{\tau}(S) f \mid R_{1-\tau}(S) g\right)_{L^{2}} \\
& =\left\langle a \circ S, W_{\tau}\left(R_{\tau}(S) f, R_{1-\tau}(S) g\right)\right\rangle \\
& =\left\langle a, W_{\tau}\left(R_{\tau}(S) f, R_{1-\tau}(S) g\right) \circ S^{-1}\right\rangle
\end{aligned}
$$

(the last identity by using the change of variables $z \longmapsto S^{-1} z$ and the fact that $\operatorname{det} S=1$ ). Formula (3.14) follows.

Notice that we recover the usual symplectic covariance formula (3.13) for the cross-Wigner transform when $\tau=\frac{1}{2}$. 


\subsection{The operators $\boldsymbol{R}_{\tau}(S)$ as pseudo-differential operators.}

3.2.1. The twisted symbol of $R_{\tau}(S)$. The following result identifies $R_{\tau}(S)$ as a $\tau$ pseudo-differential operator:

Proposition 5. Let $S \in \operatorname{Sp}_{(0)}(2 n, \mathbb{R})$. We have

$$
R_{\tau}(S)=\int r_{\sigma}(z) \widehat{T}_{\tau}(z) d z,
$$

where the twisted symbol $r_{\sigma}$ is given by the formula

$$
r_{\sigma}(z)=\frac{1}{\sqrt{|\operatorname{det}(S-I)|}} e^{i \pi M(S) z^{2}} .
$$

Proof. We have (see the proof of Proposition [3)

$$
R_{\tau}(S)=\int e^{i \pi \sigma(S z, z)} \widehat{T}_{\tau}((S-I) z) d z .
$$

The change of variables $z^{\prime}=(S-I) z$ yields

$$
\Gamma_{\tau}(S)=|\operatorname{det}(S-I)|^{-1} \int e^{i \pi \sigma\left(S(S-I)^{-1} z^{\prime}, z^{\prime}\right)} \widehat{T}_{\tau}\left(z^{\prime}\right) d z^{\prime} .
$$

Since $S(S-I)^{-1}=I+(S-I)^{-1}$ we have

$$
\begin{aligned}
\sigma\left(S(S-I)^{-1} z^{\prime}, z^{\prime}\right) & =\sigma\left((S-I)^{-1} z^{\prime}, z^{\prime}\right) \\
& =J(S-I)^{-1} z^{\prime} \cdot z^{\prime} \\
& =\left(\frac{1}{2} J+J(S-I)^{-1}\right) z^{\prime} \cdot z^{\prime} \\
& =M(S) z^{\prime} \cdot z^{\prime}
\end{aligned}
$$

and hence the expression (3.16) for $r_{\sigma}(z)$.

3.2.2. Example: The Fourier transform. For practical calculations the kernel formula (2.11) is useful. It immediately yields

$$
K_{\tau}(S)(x+y, y)=\frac{1}{\sqrt{|\operatorname{det}(S-I)|}} \int e^{2 \pi i(\tau x+y) p} e^{i \pi M(S) z^{2}} d p .
$$

This formula can be used for the calculation of explicit expressions for the operators $R_{\tau}(S)$. Let us give an example. Choosing $S=J$ we have $M(S)=\frac{1}{2} I$; a straightforward computation yields

$$
K_{\tau}(J)(x, y)=e^{i \frac{n \pi}{4}} e^{\frac{i \pi}{2}(x-y)^{2}} e^{-2 \pi i(\tau x+(1-\tau) y)^{2}} .
$$

Notice that when $\tau=\frac{1}{2}$ we get

$$
K_{1 / 2}(J)(x, y)=e^{i \frac{n \pi}{4}} e^{-2 \pi x y} .
$$

Hence $R_{1 / 2}(J)$ is, up to a factor, the usual Fourier transform. In fact we have

$$
R_{1 / 2}(J) \in \operatorname{Mp}(2 n, \mathbb{R}) ;
$$

$R_{1 / 2}(J)$ is the metaplectic operator $\widehat{J}$ with projection $J$ on the symplectic group (see e.g. de Gosson [12, 16], Folland [8]). This is not pure coincidence, as we will see in a moment when we discuss the metaplectic group. 
3.2.3. Application to Kohn-Nirenberg operators. Kohn-Nirenberg operators are widely used in time-frequency analysis. They are called normally ordered operators in quantum mechanics, and standard pseudo-differential operators in microlocal analysis, where they have been an important tool since the early 1970s. They correspond to Shubin operators with the choice $\tau=1$, leading via formula (1.2) of the Introduction to the ordering

$$
x_{j}^{m} p_{j}^{\ell} \stackrel{\mathrm{KN}}{\longrightarrow} \widehat{X}_{j}^{m} \widehat{P}_{j}^{\ell}
$$

for monomial symbols. More generally, in view of (2.2), Kohn-Nirenberg operators $A_{1}=a(x, D)$ are formally given by the expression

$$
a(x, D) f(x)=\int e^{-2 \pi i p x} a(x, p) \widehat{f}(p) d p
$$

where $\widehat{f}=F f$ is the Fourier transform of $f$; the function $a$ is usually referred to as the Kohn-Nirenberg symbol of $a(x, D)$. The formula above actually makes sense as soon as $f \in \mathcal{S}\left(\mathbb{R}^{n}\right)$ and $a \in L^{\infty}\left(\mathbb{R}^{2 n}\right)$ (this latter condition will be satisfied in what follows), but one can give a meaning to (3.21) for much larger classes of symbols (e.g. the Hörmander classes [19]). Let us identify the intertwining operator $R_{1}(S)$.

Proposition 6. Assume that $\operatorname{det}(S \pm I) \neq 0$. The Kohn-Nirenberg symbol of the operator $R_{1}(S)$ is

$$
r(z)=2^{-n / 2} e^{\frac{i \pi}{4} \operatorname{sign} M(S)} \frac{1}{\sqrt{|\operatorname{det}(S+I)|}} e^{4 i \pi M(-S) z^{2}}
$$

where $M(-S)=J M(S) J$. Conversely, every function $r(z)=C e^{4 i \pi M z^{2}}$ with $M$ real symmetric and $\operatorname{det}\left(M-\frac{1}{2} J\right) \neq 0$ is such that

$$
r(x, D) a(x, D)=(a \circ S)(x, D) r(x, D),
$$

where $S=\left(M-\frac{1}{2} J\right)^{-1}\left(M+\frac{1}{2} J\right)$.

Proof. Since the symplectic Fourier transform $F_{\sigma}$ is involutive, we have $r(z)=$ $F_{\sigma} r_{\sigma}(z)=F r_{\sigma}(J z)$. Using the Fresnel formula (3.5) we obtain after a few straightforward calculations

$$
r(z)=2^{-n / 2} e^{\frac{i \pi}{4} \operatorname{sign} M(S)} \frac{1}{\sqrt{|\operatorname{det}(S+I)|}} e^{i \pi J M(S)^{-1} J z \cdot z} .
$$

Using definition (3.1) of the symplectic Cayley transform we see that $J M(S)^{-1} J=$ $4 M(-S)$, hence formula (3.22). The converse property is obvious, taking property (3.2) of the symplectic Cayley transform into account.

As an illustration of (3.22) we see that the Kohn-Nirenberg symbol $j(z)$ of the Fourier transform leads, in the case of odd $n=2 k+1$, to the simple formula

$$
j(z)=2^{-(k+1)}(-i)^{k} e^{-2 \pi i z^{2}} .
$$

In the case of even $n$ we have $\operatorname{det}(J+I)=0$, so formula (3.22) does not apply. 
3.2.4. Discussion of the non-unitarity of $R_{\tau}(S)$. The relations (2.8)-(2.9) satisfied by the operators $\widehat{T}_{\tau}(z)$ are the same as those satisfied by the usual Heisenberg operators $\widehat{T}(z)$. Now recall how the existence of the "metaplectic representation" is often justified in the literature (see, e.g., Folland [8], §4.2, Reiter [22], Stein [24], §12.7). We first remark that the formula $\rho(z, t)=e^{2 \pi i t} \widehat{T}(z)$ defines a unitary and irreducible representation (the "Schrödinger representation") of the Heisenberg group $\mathrm{H}(2 n)$, which is $\mathbb{R}^{2 n} \times \mathbb{R}$ equipped with the group law

$$
(z, t)\left(z^{\prime}, t^{\prime}\right)=\left(z+z^{\prime}, t+t^{\prime}+\frac{1}{2} \sigma\left(z, z^{\prime}\right)\right) .
$$

For $S \in \operatorname{Sp}(2 n, \mathbb{R})$ define $\rho^{S}(z, t)=\rho(S z, t) ; \rho^{S}$ is also a representation of $\mathrm{H}(2 n)$, and since $\rho^{S}$ and $\rho$ are the same on the center $\{(0, t): t \in \mathbb{R}\}$ of $\mathrm{H}(2 n)$ they are equivalent. Hence there exists a unitary operator $U_{S}$ on $L^{2}\left(\mathbb{R}^{n}\right)$ such that $U_{S} \widehat{T}(z)=\widehat{T}(S z) U_{S}$. Since the representation $\rho$ is irreducible, it follows that this formula defines the operator $U_{S}$ uniquely up to a constant multiple with modulus one (Stone-von Neumann's theorem). The operators $U_{S}$ do not lead to a representation of $\operatorname{Sp}(2 n, \mathbb{R})$ because $U_{S S^{\prime}}=c\left(S, S^{\prime}\right) U_{S} U_{S^{\prime}}$, where $c$ is a non-trivial cocycle. They do thus only lead to a projective representation of the symplectic group. Using standard group cohomology one then shows however that there exists a function $\mu(S): \operatorname{Sp}(2 n, \mathbb{R}) \longrightarrow \mathbb{C}$ such that the operators $\widetilde{S}=\mu(S) U_{S}$ form a projective representation of the universal covering $\pi: \widetilde{\mathrm{Sp}}(2 n, \mathbb{R}) \longrightarrow \mathrm{Sp}(2 n, \mathbb{R})$, which descends to a unitary representation of the double covering $\pi: \operatorname{Sp}_{2}(2 n, \mathbb{R}) \longrightarrow \operatorname{Sp}(2 n, \mathbb{R})$. This is the metaplectic group $\operatorname{Mp}(2 n, \mathbb{R})$. (The index $\mu(S)$ is related to the Maslov index; it has been studied in detail in de Gosson [9, 10, 12, 16, following earlier work of Leray [21.) The reason for which the operators $R_{\tau}(S)$ are not unitarily equivalent to the elements of $\operatorname{Mp}(2 n, \mathbb{R})$ comes precisely from the fact that the $R_{\tau}(S)$ are not unitary (unless $\tau=\frac{1}{2}$; recall from Proposition 3 that $R_{\tau}(S)^{*}=R_{1-\tau}(S)^{-1}$ ). Therefore, these operators can, at best, generate a projective representation of the symplectic group. We will show in the next section that it is however possible to construct a group of non-unitary operators having properties similar to those of the metaplectic group $\operatorname{Mp}(2 n, \mathbb{R})$. However, this will need a careful study of the generators of the latter, following some of our previous work.

3.3. The issue of $L^{2}$-boundedness: A partial result. We will prove that each operator $R_{\tau}(S)$ is bounded on $L^{2}\left(\mathbb{R}^{n}\right)$ provided that the parameter $\tau$ is sufficiently close to the "Weyl value" $\frac{1}{2}$. Intuitively speaking, it means that such operators are small deformations of the corresponding metaplectic operator $\widehat{S}$ (see the description of $\operatorname{Mp}(2 n, \mathbb{R})$ in the next section) and therefore bounded on the square integrable functions. This partial result is in accordance with the theory of the " $\varepsilon$-Wigner transform"

$$
W^{(\varepsilon)}(f, g)=\frac{1}{2 \varepsilon} \int_{\frac{1}{2}-\varepsilon}^{\frac{1}{2}+\varepsilon} W_{\tau}(f, g) d \tau,
$$

developed by Boggiatto et al. in [2, where they show that $W^{(\varepsilon)}(f, g)$ has properties similar to those of $W(f, g)$ as $\varepsilon \rightarrow 0^{+}$.

Proposition 7. Let $S \in \operatorname{Sp}_{(0)}(2 n, \mathbb{R})$. There exists $\varepsilon>0$ such that $R_{\tau}(S)$ : $L^{2}\left(\mathbb{R}^{n}\right) \longrightarrow L^{2}\left(\mathbb{R}^{n}\right)$ for $\frac{1}{2}-\varepsilon \leq \tau \leq \frac{1}{2}+\varepsilon$, and there exists a constant $C(\varepsilon, S)$ such 
that

$$
\left\|R_{\tau}(S) f\right\|_{L^{2}} \leq C(\varepsilon, S)\|f\|_{L^{2}}
$$

for all $f \in L^{2}\left(\mathbb{R}^{n}\right)$.

Proof. We begin by noting that formulae (3.15), (3.16) in Proposition 5 can be rewritten, using (2.6), as

$$
\begin{aligned}
R_{\tau}(S) & =\frac{1}{\sqrt{|\operatorname{det}(S-I)|}} \int e^{i \pi M(S) z^{2}} e^{i \pi(2 \tau-1) p x} \widehat{T}(z) d z \\
& =\frac{1}{\sqrt{|\operatorname{det}(S-I)|}} \int e^{i \pi M^{\prime} z^{2}} \widehat{T}(z) d z,
\end{aligned}
$$

where the symmetric matrix $M^{\prime}$ is given by

$$
M^{\prime}=M+\left(\tau-\frac{1}{2}\right) N, N=\left(\begin{array}{ll}
0 & I \\
I & 0
\end{array}\right) .
$$

By definition of the symplectic Cayley transform we have

$$
M(S)=\frac{1}{2} J+J(S-I)^{-1}
$$

(formula (3.2)); hence $\operatorname{det}\left(M(S)-\frac{1}{2} J\right) \neq 0$. It follows from the continuity of the determinant function that we will have $\operatorname{det}\left(M^{\prime}-\frac{1}{2} J\right) \neq 0$ as well if $\tau-\frac{1}{2}$ is chosen small enough, and in this case $M^{\prime}=M\left(S^{\prime}\right)$ for some $S^{\prime} \in \operatorname{Sp}_{(0)}(2 n, \mathbb{R})$. We can thus write

$$
R_{\tau}(S)=\frac{1}{\sqrt{|\operatorname{det}(S-I)|}} \int e^{i \pi M\left(S^{\prime}\right) z^{2}} \widehat{T}(z) d z
$$

and hence

$$
R_{\tau}(S)=\sqrt{\left|\operatorname{det}\left(S^{\prime}-I\right)\right|} R_{1 / 2}\left(S^{\prime}\right),
$$

so $R_{\tau}(S)$ is a multiple of a metaplectic operator. Formula (3.26) follows.

It would certainly be interesting to see whether the result above can be improved. We note that formula (3.28) shows explicitly why the operators $R_{\tau}(S)$ are generally not unitary.

\section{The GROuP $\operatorname{Mp}_{\tau}(2 n, \mathbb{R})$}

\subsection{The metaplectic group $\operatorname{Mp}(2 n, \mathbb{R})$.}

4.1.1. Definition in terms of quadratic Fourier transforms. We recall that the metaplectic group $\operatorname{Mp}(2 n, \mathbb{R})$ is a faithful unitary representation in $L^{2}\left(\mathbb{R}^{n}\right)$ of the double cover $\pi: \operatorname{Sp}_{2}(2 n, \mathbb{R}) \longrightarrow \mathrm{Sp}(2 n, \mathbb{R})$ of the symplectic group. One of the possible definitions of $\operatorname{Mp}(2 n, \mathbb{R})$ is the following (Leray [21, de Gosson 10, 12, 16]): it is the group generated by the operators $\widehat{S}_{W, m}: \mathcal{S}\left(\mathbb{R}^{n}\right) \longrightarrow \mathcal{S}\left(\mathbb{R}^{n}\right)$ with distributional kernel

$$
K_{W, m}\left(x, x^{\prime}\right)=i^{m-n / 2} \sqrt{\left|\operatorname{det} B^{-1}\right|} e^{2 \pi i W\left(x, x^{\prime}\right),}
$$

where $W\left(x, x^{\prime}\right)$ (the "generating quadratic form") and $B$ are defined as follows. If $S_{W}=\left(\begin{array}{ll}A & B \\ C & D\end{array}\right)$ is symplectic with $\operatorname{det} B \neq 0$, then

$$
W\left(x, x^{\prime}\right)=\frac{1}{2} D B^{-1} x^{2}-B^{-1} x \cdot x^{\prime}+\frac{1}{2} B^{-1} A x^{\prime 2} ;
$$


the integer $m$ (the "Maslov index") is defined by

$$
m \pi \equiv \arg \operatorname{det} B \bmod 2 \pi .
$$

The operator $\widehat{S}_{W, m}$ is invertible with inverse

$$
\widehat{S}_{W, m}^{-1}=\widehat{S}_{W^{*}, m^{*}}, W^{*}\left(x, x^{\prime}\right)=-W\left(x^{\prime}, x\right), m^{*}=n-m .
$$

Notice that the condition $\operatorname{det} B \neq 0$ means that $S_{W}$ is a free symplectic matrix; equivalently $S\left(0 \times \mathbb{R}^{n}\right) \cap\left(0 \times \mathbb{R}^{n}\right)=0$. The quadratic form $W$ is a generating function for $S$; that is, $(x, p)=S\left(x^{\prime}, p^{\prime}\right)$ is equivalent to $p=\partial_{x} W\left(x, x^{\prime}\right)$ and $p^{\prime}=-\partial_{x^{\prime}} W\left(x, x^{\prime}\right)$. We notice, for further use, that the operator $\widehat{S}_{W, m}$ can be written as a product of elementary unitary operators:

$$
\widehat{S}_{W, m}=\widehat{V}_{-D B^{-1}} \widehat{M}_{B^{-1}, m} \widehat{J V}_{-B^{-1} A},
$$

where $\widehat{J}=e^{-i \frac{n \pi}{4}} F$ and

$$
\widehat{V}_{-D B^{-1}} f(x)=e^{i \pi D B^{-1} x^{2}} f(x), \widehat{M}_{B^{-1}, m} f(x)=i^{m} \sqrt{\left|\operatorname{det} B^{-1}\right|} f\left(B^{-1} x\right) .
$$

Observe that $D B^{-1}$ and $B^{-1} A$ are symmetric matrices because $S$ is symplectic. Projecting on $\operatorname{Sp}(2 n, \mathbb{R})$, formula (4.5) yields the matrix factorization

$$
S_{W}=V_{-D B^{-1}} M_{B^{-1}} J V_{-B^{-1} A}
$$

with

$$
V_{-D B^{-1}}=\left(\begin{array}{cc}
I & 0 \\
D B^{-1} & I
\end{array}\right) \quad, M_{B^{-1}}=\left(\begin{array}{cc}
B & 0 \\
0 & \left(B^{T}\right)^{-1}
\end{array}\right) .
$$

The matrices $V_{-P}, M_{L}$ generate, together with $J$, the symplectic group, and the corresponding operators $\widehat{V}_{-P}, \widehat{M}_{L, m}, \widehat{J}$ generate the group $\operatorname{Mp}(2 n, \mathbb{R})$.

4.1.2. A factorization result. An essential (and not so well-known) fact (Leray [21]) is that every $\widehat{S} \in \operatorname{Mp}(2 n, \mathbb{R})$ can be written as the product $\widehat{S}_{W, m} \widehat{S}_{W^{\prime}, m^{\prime}}$ of two metaplectic operators with kernel of the type (4.1). It is related to the fact that every $S \in \operatorname{Sp}(2 n, \mathbb{R})$ can be written as a product $S_{W} S_{W^{\prime}}$ of two free symplectic matrices (de Gosson [10, 12, 16]). These factorizations are of course non-unique. We will need the following refinement of this property:

Lemma 4.1. The generating quadratic forms $W$ and $W^{\prime}$ in the factorizations $\widehat{S}=$ $\widehat{S}_{W, m} \widehat{S}_{W^{\prime}, m^{\prime}}, S=S_{W} S_{W^{\prime}}$ can be chosen in such a way that $\operatorname{det}\left[\left(S_{W}-I\right)\left(S_{W^{\prime}}-I\right)\right]$ $\neq 0$, i.e. $S_{W}, S_{W^{\prime}} \in \mathrm{Sp}_{(0)}(2 n, \mathbb{R})$.

Proof. We first remark that

$$
\operatorname{det}\left(S_{W}-I\right)=\operatorname{det}(-B) \operatorname{det}\left[B^{-1} A+D B^{-1}-B^{-1}-\left(B^{T}\right)^{-1}\right]
$$

(Lemma 4 in de Gosson [11]). The next step consists in remarking that in view of the factorization (4.5) we have

$$
\widehat{S}_{W, m} \widehat{S}_{W^{\prime}, m^{\prime}}=\widehat{V}_{-P} \widehat{M}_{B^{-1}, m} \widehat{J V} \widehat{V}_{-\left(P^{\prime}+Q\right)} \widehat{M}_{\left(B^{\prime}\right)^{-1}, m^{\prime}} \widehat{J V}_{-Q^{\prime}}
$$

with $P=D B^{-1}, P^{\prime}=D^{\prime}\left(B^{\prime}\right)^{-1}, Q=B^{-1} A, Q^{\prime}=\left(B^{\prime}\right)^{-1} A^{\prime}$. We next remark that the right-hand side of (4.10) remains unchanged if we replace $P^{\prime}$ with $P^{\prime}+\lambda I$ and $Q$ with $Q^{\prime}-\lambda I$. Choosing $\lambda$ so that

$$
\begin{array}{r}
\operatorname{det}\left[P+Q-\lambda I+B^{-1}-\left(B^{T}\right)^{-1}\right] \neq 0, \\
\operatorname{det}\left[P^{\prime}+Q^{\prime}+\lambda I-\left(B^{\prime}\right)^{-1}-\left(B^{\prime T}\right)^{-1}\right] \neq 0,
\end{array}
$$


we have $\operatorname{det}\left(S_{W}-I\right) \neq 0$ and $\operatorname{det}\left(S_{W^{\prime}}-I\right) \neq 0$ in view of the equality (4.9). The lemma follows by replacing, if necessary, $W\left(x, x^{\prime}\right)$ with $W\left(x, x^{\prime}\right)-\frac{1}{2} \lambda x^{2}$ and $W^{\prime}\left(x, x^{\prime}\right)$ with $W^{\prime}\left(x, x^{\prime}\right)+\frac{1}{2} \lambda x^{\prime 2}$.

We remark that the symmetric matrix

$$
W^{\prime \prime}=B^{-1} A+D B^{-1}-B^{-1}-\left(B^{T}\right)^{-1}
$$

is the Hessian matrix of the generating quadratic form $W$.

4.2. The group $\mathrm{Mp}_{\tau}(2 n, \mathbb{R})$. In de Gosson [1] (also see [12, 13, 14, 16]) we proved the following result:

Proposition 8. Let $\widehat{S} \in \operatorname{Mp}(2 n, \mathbb{R})$ have the projection $S \in \operatorname{Sp}_{(0)}(2 n, \mathbb{R})$. Then

$$
\widehat{S}=i^{\nu(\widehat{S})} \sqrt{|\operatorname{det}(S-I)|} \int \widehat{T}(S z) \widehat{T}(-z) d z,
$$

where the integer $\nu(\widehat{S})$ corresponds to the choice (modulo 2) of an argument of $\operatorname{det}(S-I)$ :

$$
\nu(\widehat{S}) \pi \equiv n \pi+\arg \operatorname{det}(S-I) \bmod 2 \pi .
$$

When $S=S_{W}$ is a free symplectic matrix we have

$$
\nu\left(\widehat{S}_{W, m}\right) \equiv m-\text { Inert } W^{\prime \prime} \bmod 2,
$$

where Inert $W^{\prime \prime}$ is the number of negative eigenvalues of the Hessian matrix (4.11).

It follows that our operators $R_{\tau}(S)$ coincide, up to a unimodular factor, with metaplectic operators when $\tau=\frac{1}{2}$ :

Corollary 1. For $S \in \operatorname{Sp}_{(0)}(2 n, \mathbb{R})$ the operators $R(S)=R_{1 / 2}(S)$ are, up to a unimodular factor $i^{\nu(S)}$, elements of the metaplectic group $\operatorname{Mp}(2 n, \mathbb{R})$.

Proof. In [14, 15] we have shown that

$$
R^{\nu}(S)=\frac{i^{\nu(S)}}{\sqrt{|\operatorname{det}(S-I)|}} \int e^{i \pi M(S) z^{2}} \widehat{T}(z) d z,
$$

where $\nu(S)$ is the Conley-Zehnder [5] index (which we describe below). The result follows since $\widehat{T}_{1 / 2}(z)=\widehat{T}(z)$.

The Conley-Zehnder index $i_{\mathrm{CZ}}(\widetilde{S})$ is, loosely speaking, a Maslov-type intersection index measuring the number of intersections of a symplectic path $\widetilde{S}: t \longmapsto S_{t}$, $0 \leq t \leq 1$, joining the identity to $S \in \operatorname{Sp}_{(0)}(2 n, \mathbb{R})$ with the variety $\{S: \operatorname{det}(S-I)=$ $0\}$, which plays the role of a "caustic". More precisely, $i_{\mathrm{CZ}}(\widetilde{S})$ associates to the path $t \longmapsto S_{t}$ an integer which only depends on the homotopy class (with fixed endpoints) of that path, and such that

$$
\operatorname{sign} \operatorname{det}(S-I)=(-1)^{n-i_{\mathrm{CZ}}(\widetilde{S})} .
$$

Equivalently,

$$
\frac{1}{\pi} \arg \operatorname{det}(S-I) \equiv n-i_{\mathrm{CZ}}(\widetilde{S}) \bmod 2 .
$$

In [11] we have proven that when $S$ is a free symplectic matrix $S_{W}$, then

$$
i_{\mathrm{CZ}}\left(\widetilde{S_{W}}\right) \equiv m+n-\text { Inert } W^{\prime \prime} \bmod 2 \text {. }
$$


For a detailed discussion of that index (including an extension to the case $\operatorname{det}(S-$ $I)=0$ and its expression in terms of the Leray-Maslov index [21]) see our paper [15.

In analogy with the definition of $\operatorname{Mp}(2 n, \mathbb{R})$ in terms of the basic metaplectic operators $\widehat{S}_{W, m}$ we define a group $\operatorname{Mp}_{\tau}(2 n, \mathbb{R})$ of (non-unitary) operators as follows. Let $S_{W} \in \operatorname{Sp}_{(0)}(2 n, \mathbb{R})$ be a free symplectic matrix; we know from Lemma 4.1 that every $S \in \operatorname{Sp}(2 n, \mathbb{R})$ is the product of two such matrices. Let $\widehat{S}_{W, m} \in \operatorname{Mp}(2 n, \mathbb{R})$ be any one of the two metaplectic operators covering $S_{W}$. We now define

$$
\begin{gathered}
R_{\tau}^{\nu\left(S_{W}\right)}\left(S_{W}\right)=i^{\nu\left(S_{W}\right)} R_{\tau}\left(S_{W}\right), \\
\nu\left(S_{W}\right) \equiv n \pi+\arg \operatorname{det}(S-I) \bmod 2 \pi .
\end{gathered}
$$

The operators $R_{\tau}^{\nu\left(S_{W}\right)}\left(S_{W}\right)$ are invertible, and

$$
R_{\tau}^{\nu\left(S_{W}\right)}\left(S_{W}\right)^{-1}=R_{\tau}^{-\nu\left(S_{W}\right)}\left(S_{W}^{-1}\right)=R_{\tau}^{-\nu\left(S_{W}\right)}\left(S_{W^{*}}\right)
$$

(the first equality in view of formula (3.10) in Proposition 3 , and the second since the free symplectic matrix $S_{W}^{-1}$ is generated by $W^{*}\left(x, x^{\prime}\right)=-W\left(x^{\prime}, x\right)$; cf. (4.4) ).

By definition $\operatorname{Mp}_{\tau}(2 n, \mathbb{R})$ is the group of operators $\mathcal{S}\left(\mathbb{R}^{n}\right) \longrightarrow \mathcal{S}\left(\mathbb{R}^{n}\right)$ generated by $R_{\tau}^{\nu\left(S_{W}\right)}\left(S_{W}\right)$ : for an arbitrary $S \in \operatorname{Sp}(2 n, \mathbb{R})$ the operator $R_{\tau}^{\nu}(S) \in \operatorname{Mp}_{\tau}(2 n, \mathbb{R})$ is the product of a finite number of $R_{\tau}^{\nu\left(S_{W}\right)}\left(S_{W}\right)$ with $S_{W} \in \operatorname{Sp}_{(0)}(2 n, \mathbb{R})$. We have moreover the following equivalence of the fact that each $\widehat{S} \in \operatorname{Mp}(2 n, \mathbb{R})$ can be written as the product of two metaplectic operators of the type $\widehat{S}_{W, m}$ :

Proposition 9. Let $S \in \operatorname{Sp}_{(0)}(2 n, \mathbb{R})$ and choose $S_{W}, S_{W^{\prime}} \in \operatorname{Sp}_{(0)}(2 n, \mathbb{R})$ such that $S=S_{W} S_{W^{\prime}}$. We have

$$
R_{\tau}^{\nu\left(S_{W}\right)}\left(S_{W}\right) R_{\tau}^{\nu\left(S_{W^{\prime}}\right)}\left(S_{W^{\prime}}\right)=R_{\tau}^{\nu(S)}\left(S_{W} S_{W^{\prime}}\right),
$$

where the index $\nu(S)$ is given by the formula

$$
\nu(S)=\nu\left(S_{W}\right)+\nu\left(S_{W^{\prime}}\right)+\frac{1}{2} \operatorname{sign} M\left(S S^{\prime}\right) .
$$

Proof. It follows from formula (3.9) in Proposition 3 that we have

$$
\begin{aligned}
& R_{\tau}^{\nu\left(S_{W}\right)}\left(S_{W}\right) R_{\tau}^{\nu\left(S_{W^{\prime}}\right)}\left(S_{W^{\prime}}\right)=e^{i \Phi} R_{\tau}(S) R_{\tau}\left(S^{\prime}\right), \\
& \Phi=\frac{\pi}{2}\left(\nu\left(S_{W}\right)+\nu\left(S_{W^{\prime}}\right)+\frac{1}{2} \operatorname{sign} M\left(S_{W} S_{W^{\prime}}\right)\right),
\end{aligned}
$$

hence the assertion.

Proposition 10. For every $R_{\tau}^{\nu}(S) \in \mathrm{Mp}_{\tau}(2 n, \mathbb{R})$ we have the symplectic covariance formulae

$$
R_{\tau}^{\nu}(S) \mathrm{Op}_{\tau}(a)=\mathrm{Op}_{\tau}(a \circ S) R_{\tau}^{\nu}(S)
$$

and, for $f, g \in \mathcal{S}\left(\mathbb{R}^{n}\right)$,

$$
W_{\tau}\left(R_{\tau}(S) f, R_{1-\tau}(S) g\right)(z)=W_{\tau}(f, g)\left(S^{-1} z\right) .
$$

Proof. The intertwining formula (4.24) follows from Proposition 3 (formula (3.8)), and formula (4.25) follows from Proposition 4 (formula (3.14)). 


\section{The CASE OF BORN-JoRdAN OPERATORS}

\subsection{Born-Jordan operators.}

5.1.1. Motivations. Concurrently with Weyl, the physicists Born and Jordan 4 ] elaborated on Heisenberg's seminal paper [18] on "matrix mechanics" and proposed the quantization rule

$$
x_{j}^{m} p_{j}^{\ell} \stackrel{\mathrm{BJ}}{\longrightarrow} \frac{1}{\ell+1} \sum_{k=0}^{\ell}{\widehat{P_{j}}}^{\ell-k} \widehat{X}_{j}^{m}{\widehat{P_{j}}}^{k}
$$

which coincides with (1.1) when $m+\ell \leq 2$. We now make the following fundamental remark: the Born-Jordan prescription (5.1) is obtained by averaging the $\tau$-ordering (1.2) on the interval $[0,1]$; this is immediately seen using the property

$$
B(k+1, \ell-k+1)=\int_{0}^{1}(1-\tau)^{k} \tau^{\ell-k} d \tau=\frac{k !(\ell-k) !}{(k+\ell+1) !}
$$

of the beta function. On the other hand, as already mentioned, Boggiatto and his collaborators have indirectly rediscovered the Born-Jordan prescription by introducing in [1, via the $\tau$-Wigner function, the pseudo-differential operators

$$
A_{\mathrm{BJ}}=\int_{0}^{1} A_{\tau} d \tau \text {. }
$$

These facts show that this scheme certainly deserves to be studied, both from a quantum mechanical and a time-frequency analysis perspective.

5.1.2. Definition of Born-Jordan operators. In [1] Boggiatto et al. (also see [2, 3]) define a transform $Q: \mathcal{S}\left(\mathbb{R}^{n}\right) \times \mathcal{S}\left(\mathbb{R}^{n}\right) \longrightarrow \mathcal{S}\left(\mathbb{R}^{n}\right)$ by integrating over $[0,1]$ the $\tau$-cross Wigner transforms (2.15). We will use the notation $Q=W_{\mathrm{BJ}}$; thus, for $f, g \in \mathcal{S}\left(\mathbb{R}^{n}\right)$,

$$
W_{\mathrm{BJ}}(f, g)=\int_{0}^{1} W_{\tau}(f, g) d \tau .
$$

They show that $W_{\mathrm{BJ}}(f, g)$ has properties similar to those of the usual cross-Wigner transform, and that it belongs to the Cohen class. In particular, $W_{\mathrm{BJ}} f=W_{\mathrm{BJ}}(f, f)$ satisfies the marginal properties

$$
\int W_{\mathrm{BJ}} f(x, p) d p=|f(x)|^{2}, \int W_{\mathrm{BJ}} f(x, p) d x=|F f(p)|^{2}
$$

for every $f \in L^{1}\left(\mathbb{R}^{n}\right) \cap L^{2}\left(\mathbb{R}^{n}\right)$ (this immediately follows from property (2.16) of the $\tau$-Wigner transform). We refer to 1 for a discussion of the advantages of using $W_{\mathrm{BJ}}(f, g)$ instead of $W(f, g)$ in time-frequency analysis.

For $a \in \mathcal{S}^{\prime}\left(\mathbb{R}^{n}\right)$ we can define an operator, which we denote $A_{\mathrm{BJ}}$, by the formula

$$
\left(A_{\mathrm{BJ}} f \mid g\right)_{L^{2}}=\left\langle a, W_{\mathrm{BJ}}(f, g)\right\rangle
$$

$f, g \in \mathcal{S}\left(\mathbb{R}^{n}\right)$ (cf. (2.14) $)$. We will call $A_{\mathrm{BJ}}$ the Born-Jordan operator with symbol $a$ and write $A_{\mathrm{BJ}}=\mathrm{Op}_{\mathrm{BJ}}(a)$.

Proposition 11. The Born-Jordan operator $A_{B J}=\mathrm{Op}_{\mathrm{BJ}}(a)$ is given by

$$
A_{B J}=\int a_{\sigma}(z) \Theta(z) \widehat{T}(z) d z
$$


where $\Theta$ is the real function defined by

$$
\Theta(z)=\frac{\sin (2 \pi p x)}{2 \pi p x} .
$$

The operator $A_{B J}$ is a continuous operator $\mathcal{S}\left(\mathbb{R}^{n}\right) \longrightarrow \mathcal{S}^{\prime}\left(\mathbb{R}^{n}\right)$ for every a $\in \mathcal{S}^{\prime}\left(\mathbb{R}^{2 n}\right)$. That this formula really defines a continuous operator $\mathcal{S}\left(\mathbb{R}^{n}\right) \longrightarrow \mathcal{S}^{\prime}\left(\mathbb{R}^{n}\right)$ follows from the fact that $\Theta \in L^{\infty}\left(\mathbb{R}^{2 n}\right)$.

Proof. Using the representation (2.10) of the $\tau$-operators we have

$$
A_{\mathrm{BJ}}=\mathrm{Op}_{\mathrm{BJ}}(a)=\int a_{\sigma}(z) \widehat{T}_{\mathrm{BJ}}(z) d z
$$

where $\widehat{T}_{\mathrm{BJ}}(z)$ is the unitary operator defined by

$$
\widehat{T}_{\mathrm{BJ}}(z)=\int_{0}^{1} \widehat{T}_{\tau}(z) d \tau=\frac{\sin (2 \pi p x)}{2 \pi p x} \widehat{T}(z) .
$$

Integrating both sides of formula (2.6) for $\widehat{T}_{\tau}(z)$ in the interval $[0,1]$ we have $\widehat{T}_{\mathrm{BJ}}(z)=\Theta(z) \widehat{T}(z)$, hence the expression (5.5).

In view of the relation (2.3) between a $\tau$-operator and its adjoint we have

$$
\mathrm{Op}_{\mathrm{BJ}}(a)^{*}=\mathrm{Op}_{\mathrm{BJ}}(\bar{a}) \text {. }
$$

Hence the Born-Jordan operators share with Weyl operators the property of being (formally) self-adjoint if and only if their symbol is real. This makes Born-Jordan operators good alternative candidates for quantization.

The reader is urged to notice that while every Born-Jordan operator is a Weyl operator, the converse property is not true because an arbitrary distribution $b_{\sigma} \in$ $\mathcal{S}^{\prime}\left(\mathbb{R}^{2 n}\right.$ ) cannot in general be written in the form $a_{\sigma} \Theta$ (see de Gosson and Luef [17; see also the discussion in Kauffmann [20] where conceptual questions from quantum mechanics are taken up).

5.2. Partial metaplectic covariance of Born-Jordan operators. The intertwining properties for $\tau$ operators do not carry over to the Born-Jordan case. It is meaningless to expect a relation such as $R_{\mathrm{BJ}}(S) \widehat{T}_{\mathrm{BJ}}(z)=\widehat{T}_{\mathrm{BJ}}(S z) R_{\mathrm{BJ}}(S)$ which would lead to a symplectic covariance property of the type (3.8). The good news is, however, that Born-Jordan operators enjoy a symplectic covariance property for operators belonging to a subgroup of the standard metaplectic group $\operatorname{Mp}(2 n, \mathbb{R})$. Recall that $\operatorname{Mp}(2 n, \mathbb{R})$ is generated by the modified Fourier transform $\widehat{J}=e^{i \frac{n \pi}{4}} F$, the multiplication operators $\widehat{V}_{-P} f=e^{i \pi P x^{2}} f\left(P=P^{T}\right)$ and the unitary scaling operators $\widehat{M}_{L, m} f(x)=i^{m} \sqrt{|\operatorname{det} L|} f(L x)(\operatorname{det} L \neq 0, m \pi=\arg \operatorname{det} L)$. The projections of these operators on $\operatorname{Sp}(2 n, \mathbb{R})$ are, respectively, $J, V_{-P}=\left(\begin{array}{ll}I & 0 \\ P & I\end{array}\right)$, and $M_{L}=\left(\begin{array}{cc}L^{-1} & 0 \\ 0 & L^{2}\end{array}\right)$.

Proposition 12. Let $A_{B J}=\mathrm{Op}_{\mathrm{BJ}}(a)$ with $a \in \mathcal{S}^{\prime}\left(\mathbb{R}^{2 n}\right)$. We have

$$
\widehat{S} \mathrm{Op}_{\mathrm{BJ}}(a)=\mathrm{Op}_{\mathrm{BJ}}\left(a \circ S^{-1}\right) \widehat{S}
$$

for every $\widehat{S} \in \operatorname{Mp}(2 n, \mathbb{R})$, which is a product of a (finite number) of operators $\widehat{J}$ and $\widehat{M}_{L, m}$. 
Proof. It suffices to prove formula (5.10) for $\widehat{S}=\widehat{J}$ and $\widehat{S}=\widehat{M}_{L, m}$. First let $\widehat{S}$ be an arbitrary element of $\operatorname{Mp}(2 n, \mathbb{R})$. We have

$$
\begin{aligned}
\widehat{S} \mathrm{Op}_{\mathrm{BJ}}(a) & =\int a_{\sigma}(z) \Theta(z) \widehat{S} \widehat{T}(z) d z \\
& =\left(\int a_{\sigma}(z) \Theta(z) \widehat{T}(S z) d z\right) \widehat{S},
\end{aligned}
$$

where the second equality follows from the usual symplectic covariance property $\widehat{S} \widehat{T}(z)=\widehat{T}(S z) \widehat{S}$ of the Heisenberg operators. Making the change of variables $z^{\prime}=S z$ in the integral we get, since $\operatorname{det} S=1$,

$$
\int a_{\sigma}(z) \Theta(z) \widehat{T}(S z) d z=\int a_{\sigma}\left(S^{-1} z\right) \Theta\left(S^{-1} z\right) \widehat{T}(z) d z .
$$

Now, by definition of the symplectic Fourier transform we have

$$
a_{\sigma}\left(S^{-1} z\right)=\int e^{-2 \pi i \sigma\left(S^{-1} z, z^{\prime}\right)} a\left(z^{\prime}\right) d z^{\prime}=\left(a \circ S^{-1}\right)_{\sigma}(z) .
$$

Now let $\widehat{S}=\widehat{M}_{L, m}$; we have

$$
\Theta\left(M_{L}^{-1} z\right)=\frac{\sin \left(2 \pi L p \cdot\left(L^{T}\right)^{-1} x\right)}{2 \pi L p \cdot\left(L^{T}\right)^{-1} x}=\Theta(z) .
$$

Similarly $\Theta\left(J^{-1} z\right)=\Theta(z)$; hence in both cases

$$
\begin{aligned}
\widehat{S} \mathrm{Op}_{\mathrm{BJ}}(a) & =\left(\int\left(a \circ S^{-1}\right)_{\sigma} \Theta(z) \widehat{T}(z) d z\right) \widehat{S} \\
& =\operatorname{Op}_{\mathrm{BJ}}\left(a \circ S^{-1}\right) \widehat{S}
\end{aligned}
$$

whence formula (5.10).

The proof above shows that the essential step consists in noting that $\Theta\left(S^{-1} z\right)=$ $\Theta(z)$ when $S=J$ or $S=M_{L}$. It is clear that this property fails if one takes $S=V_{P}$ with $P \neq 0$, so we cannot expect to have full symplectic covariance for Born-Jordan operators since $\operatorname{Sp}(2 n, \mathbb{R})$ is generated by the set of all matrices $J, M_{L}$ and $V_{P}$. Full symplectic covariance is excluded anyway in view of our discussion in the Introduction to this paper, since this property is characteristic of Weyl calculus, and thus cannot be satisfied by any other pseudo-differential calculus.

5.3. The case of "physical" Hamiltonians. Weyl and Born-Jordan quantization are the same for monomials $x_{j}^{m} p_{j}^{\ell}$ with $m+\ell \leq 2$. It follows that every Born-Jordan operator with polynomial symbol

$$
a(z)=Q(z)+a \cdot z+b,
$$

where $Q(z)$ is a complex quadratic form in the coordinates $x_{j}, p_{k}, a \in \mathbb{C}^{2 n}, b \in \mathbb{C}$, is symplectically covariant with respect to the full metaplectic group $\operatorname{Mp}(2 n, \mathbb{R})$.

In fact, Weyl and Born-Jordan quantization are also identical for symbols arising from physical Hamiltonians

$$
H(z)=\sum_{j=1}^{n} \frac{1}{2 m_{j}}\left(p_{j}-A_{j}(x)\right)^{2}+V(x),
$$

where $A_{j}$ and $V$ are real $C^{\infty}$ functions. This easily follows from the two observations below: any $\tau$-operator with symbol $a=a(x)$ only depending on $x$ is multiplication 
by $a(x)$ (this immediately follows from formula (2.2)); applying the same formula to the term $p_{j} A_{j}$ appearing in the expansion of $\left(p_{j}-A_{j}(x)^{2}\right.$ in (5.11) we have

$$
\mathrm{Op}_{\tau}\left(p_{j} A_{j}\right) f(x)=\frac{1}{2 \pi i}\left[(1-\tau) \partial_{x_{j}}\left(A_{j} f\right)+\tau A_{j} \partial_{x_{j}} f\right]
$$

and hence, integrating from 0 to 1 ,

$$
\mathrm{Op}_{\mathrm{BJ}}\left(p_{j} A_{j}\right)=\frac{1}{2 \pi i}\left[\partial_{x_{j}}\left(A_{j} f\right)+A_{j} \partial_{x_{j}} f\right] .
$$

Collecting these facts we get

$$
H_{\mathrm{BJ}}=\sum_{j=1}^{n} \frac{1}{2 m_{j}}\left(\frac{1}{2 \pi i} \partial_{x_{j}}-A_{j}(x)\right)^{2}+V(x),
$$

which is identical with $\widehat{H}=H_{\mathrm{Weyl}}$, as can be directly verified using the expression (1.4) for Weyl pseudo-differential operators. It follows that this operator satisfies full symplectic covariance with respect to the metaplectic group.

\section{ACKNOWLEDGEMEnts}

This work has been financed by the Austrian Research Foundation FWF (Projektnummer P20442-N13).

The author would like to take the opportunity to express his thanks to the referee for useful comments and remarks, and for having pointed out an additional reference.

\section{References}

[1] P. Boggiatto, G. De Donno, A. Oliaro. Time-Frequency Representations of Wigner Type and Pseudo-Differential Operators, Transactions of the Amer. Math. Soc., 362(9) 4955-4981 (2010) MR2645057

[2] P. Boggiatto, Bui Kien Cuong, G. De Donno, A. Oliaro. Weighted integrals of Wigner representations, J. Pseudo-Differ. Oper. Appl. 1(4) 401-415 (2010) MR2747903 (2011m:42014)

[3] P. Boggiatto, G. De Donno, A. Oliaro. Hudson Theorem for $\tau$-Wigner Transforms, Quaderni scientifici del Dipartimento di Matematica, Univeristà di Torino, Quaderno N. 1, 2010

[4] M. Born, P. Jordan. Zur Quantenmechanik, Z. Physik 34, 858-888 (1925)

[5] C. Conley, E. Zehnder. Morse-type index theory for flows and periodic solutions of Hamiltonian equations, Comm. Pure and Appl. Math. 37 (1984) 207-253 MR733717 (86b:58021)

[6] H. G. Feichtinger. On a new Segal algebra, Monatsh. Math., 92 (4), 269-289 (1981) MR643206 (83a:43002)

[7] H. G. Feichtinger. Modulation Spaces: Looking Back and Ahead. Sampl. Theory Signal Image Process. 5(2), 109-140 (2006) MR2233968 (2007j:43003)

[8] G. B. Folland. Harmonic Analysis in Phase Space. Annals of Mathematics studies, Princeton University Press, Princeton, N.J. (1989) MR.983366 (92k:22017)

[9] M. de Gosson. Maslov indices on the metaplectic group $\mathrm{Mp}(n)$, Ann. Inst. Fourier 40(3), 537-555 (1990) MR 1091832 (92e:22027)

[10] M. de Gosson. Maslov Classes, Metaplectic Representation and Lagrangian Quantization, Mathematical Research 95, Wiley VCH (1997) MR1449637(98k:58097)

[11] M. de Gosson. The Weyl Representation of Metaplectic operators, Letters in Mathematical Physics 72 129-142 (2005) MR2154859(2006h:81151)

[12] M. de Gosson. Symplectic Geometry and Quantum Mechanics, Birkhäuser, Basel, series "Operator Theory: Advances and Applications" (subseries: "Advances in Partial Differential Equations"), Vol. 166 (2006) MR2241188(2007e:81050)

[13] M. de Gosson, S. de Gosson. An extension of the Conley-Zehnder Index, a product formula and an application to the Weyl representation of metaplectic operators, J. Math. Phys., 47(12), 123506, 15 pages (2006) MR2285155(2008c:53082) 
[14] M. de Gosson. Metaplectic Representation, Conley-Zehnder Index, and Weyl Calculus on Phase Space, Rev. Math. Physics, 19(8) (2007) 1149-1188 MR2362902 (2008k:81170)

[15] M. de Gosson. On the usefulness of an index due to Leray for studying the intersections of Lagrangian and symplectic paths, J. Math. Pures Appl. 91 (2009) 598-613 MR2531557 (2010f:53145)

[16] M. de Gosson. Symplectic Methods in Harmonic Analysis and in Mathematical Physics, Birkhäuser, Basel (2011) MR2827662

[17] M. de Gosson, F. Luef. Preferred Quantization Rules: Born-Jordan vs. Weyl; Applications to Phase Space Quantization, J. Pseudo-Differ. Oper. Appl. 2(1) (2011) 115-139 MR2781145

[18] W. Heisenberg. Über quantentheoretische Umdeutung kinematischer und mechanischer Beziehungen, Zeitschrift für Physik, 33, 879-893 (1925) [English translation in: B. L. van der Waerden, editor, Sources of Quantum Mechanics, Dover Publications (1968)]

[19] L. Hörmander. The Analysis of Linear Partial Differential Operators, vol. III, SpringerVerlag, Berlin, 1985 MR2304165 (2007k:35006)

[20] S. K. Kauffmann. Unambiguous quantization from the maximum classical correspondence that is self-consistent: The slightly stronger canonical commutation rule Dirac missed, Found. Phys. 41, 805-918 (2011) MR2785351

[21] J. Leray. Lagrangian Analysis and Quantum Mechanics, a mathematical structure related to asymptotic expansions and the Maslov index (the MIT Press, Cambridge, Mass., 1981); translated from Analyse Lagrangienne RCP 25, Strasbourg Collège de France, 1976-1977 MR644633(83k:58081a)

[22] H. Reiter. Metaplectic Groups and Segal Algebras, Springer (1989) MR 1011671(91k:43005)

[23] M. A. Shubin. Pseudodifferential Operators and Spectral Theory, Springer-Verlag (1987) [original Russian edition in Nauka, Moskva (1978)]

[24] E. M. Stein. Harmonic Analysis: Real Variable Methods, Orthogonality, and Oscillatory Integrals. Princeton University Press (1993) MR1232192 (95c:42002)

[25] J. Toft. Multiplication properties in pseudo-differential calculus with small regularity on the symbols, J. Pseudo-Differ. Oper. Appl. 1, 101-138 (2010) MR.2679745 (2011h:47094)

[26] A. Weil. Sur certains groupes d'opérateurs unitaires, Acta Math. 111, 143-211 (1964); also in Collected Papers, Vol. III:1-69, Springer-Verlag, Heidelberg, 1980 MR0165033 (29:2324)

[27] H. Weyl. Quantenmechanik und Gruppentheorie, Zeitschrift für Physik 46 (1927)

[28] M. W. Wong. Weyl Transforms, Springer (1998) MR.1639461 (2000c:47098)

Fakultät für Mathematik, Numerical Harmonic Analysis Group, Universität Wien, A-1090 Vienna, Austria

E-mail address: maurice.de.gosson@univie.ac.at

$U R L:$ http://cvdegosson.webs.com/ 